\title{
Treinamento de habilidades sociais educativas parentais: comparação de procedimentos a partir do tempo de intervenção ${ }^{1}$
}

\section{Training parental educational social skills: comparison procedures from the intervention's time}

\author{
Alessandra Turini Bolsoni-Silva* \\ Universidade Estadual Paulista - UNESP, Bauru, São Paulo, Brasil \\ Laura Moreira Borelli** \\ Universidade Estadual Paulista - UNESP, Bauru, São Paulo, Brasil
}

\begin{abstract}
RESUMO
O presente estudo tem como objetivo a comparação de dois procedimentos de intervenção com pais que buscaram promover melhores interações sociais com seus filhos, por meio do treinamento e fortalecimento das habilidades sociais. Os procedimentos diferiram apenas em relação ao tempo em que foram administrados: 20 sessões aplicadas uma vez por semana a um grupo (G1), e duas vezes por semana a outro grupo (G2). Como instrumento, utilizou-se o Roteiro de Entrevista de Habilidades Sociais Educativas Parentais (RE-HSE-P). Notou-se que ambos os grupos obtiveram resultados positivos, no entanto, G1 destacou-se no aumento das habilidades sociais dos pais e das crianças e G2, na diminuição dos problemas de comportamento. Conclui-se que a intervenção de maior duração pode proporcionar a maior aquisição e consolidação de habilidades, e aponta-se para a necessidade da realização de trabalhos preventivos com a população, e de estudos comparativos para o aprimoramento de procedimentos de intervenção.
\end{abstract}

Palavras-chave: habilidades sociais educativas parentais; comparação de procedimentos; intervenção com pais.

\begin{abstract}
This study aims to compare two intervention procedures with parents seeking to promote better social interactions with their children, through training and consolidation of social skills. The procedures were different only in relation to the time in which they were administered: 20 sessions applied once week for one group (G1) and twice a week for the other group (G2). The Parental Education Social Skills Interview was applied (RE-HSE-P). It was noticed that both groups have presented positives outcomes, however G1 has presented enhanced social skills for both the parents and children and $G 2$ has presented reduction on behavior problems. It is concluded that the intervention of longer duration can provide better acquisition and consolidation of skills, and it is pointed the necessity of preventive work with the population, and comparative studies for the improvement of interventional procedures.
\end{abstract}


Keywords: parental educational social skills, procedures comparison, intervention with parents.

\section{Introdução}

Os problemas de comportamento da criança compreendem um objeto de estudo da área educacional, haja vista que sua solução depende do emprego de medidas socioeducativas - capazes de desenvolver as habilidades sociais para uma socialização na escola; e também da correlação positiva da escola em relação aos problemas de comportamento e dificuldades de aprendizagem (FEITOSA, MATOS; DEL PRETTE; DEL PRETTE, 2005).

Em uma perspectiva funcional, problemas de comportamento são entendidos como "déficits e/ou excessos comportamentais que dificultariam 0 acesso da criança a novas contingências de reforçamento, que por sua vez, facilitariam a aquisição de repertórios relevantes de aprendizagem" (BOLSONI-SILVA; DEL PRETTE, 2003, p. 94).

O surgimento e/ou a manutenção de problemas de comportamento, de acordo com Bolsoni-Silva e Marturano (2006), estariam relacionados a variáveis como: a história de vida familiar, determinadas práticas parentais, condição socioeconômica, eventos estressantes, conflitos conjugais, hereditariedade dos pais e da criança, patologia parental, características da criança e da escola. Quanto mais fatores de risco estiverem presentes maiores parecem ser os problemas de comportamento da criança.

No mesmo sentido, Pinheiro, Haase, Del Prette, Amarante e Del Prette (2006) afirmam que a maioria dos comportamentos considerados inadequados das crianças surge e é mantida, principalmente, em decorrência de déficits de habilidades sociais apresentados pelos próprios pais; prejudicando, dessa forma, a manutenção dos comportamentos de seus filhos.

Por meio de uma disciplina inconsistente, pouca interação positiva, baixo monitoramento e insuficiente supervisão das atividades da criança, os pais estimulam tais problemas e ainda contribuem para a promoção de falhas no desenvolvimento do repertório de comportamentos, os quais propiciariam interações positivas da criança com colegas e adultos (BOLSONI-SILVA; DEL PRETTE, 2002; BOLSONI-SILVA; DEL PRETTE, 2003).

Comportamentos externalizantes, tais como agredir, desobedecer etc., são considerados problemas de comportamento, e podem surgir e/ou se manter exercendo a função de contracontrole, reproduzidos dos comportamentos aversivos familiares.

De acordo com Sidman (1989/1995), quando não existe a possibilidade de fuga, ou esquiva, a criança aprende a controlar seus 
controladores, descobrindo uma maneira de acabar com as punições ou ameaças.

Buscando caracterizar as queixas de pais, mães e educadores que buscam atendimento em um Centro de Psicologia Aplicada, com a intenção de melhorar seu relacionamento com seus filhos, BolsoniSilva, Paiva e Barbosa (2009) realizaram um estudo levantando dados de 2004 à 2006, com base em uma Entrevista Clínica Semiestruturada. Encontraram como queixas mais frequentes os comportamentos externalizantes da criança, e também do adolescente, tais como: agressividade, desobediência e comportamento de birra; e dificuldades dos pais/ cuidadores, tais como: gritar ou bater quando o filho emitia comportamentos considerados indesejáveis, discordância entre pai e mãe em relação à educação dos filhos, falta de consistência diante dos comportamentos do filho, dificuldades na comunicação e em estabelecer limites (BOLSONI-SILVA; PAIVA; BARBOSA, 2009).

Habilidades sociais, segundo Del Prette e Del Prette (1999; 2009), são o conjunto de desempenhos (componentes comportamentais, cognitivo-afetivos e fisiológicos), ou uma classe de respostas, apresentados pelo indivíduo para lidar de maneira adequada com as demandas de uma situação interpessoal, que inclui variáveis da cultura e contribuem para a competência social. Sua aquisição envolve um processo de aprendizagem contínuo, através das demandas próprias do estágio de desenvolvimento do indivíduo e o desempenho interpessoal apresentado. Do ponto de vista da Análise do Comportamento, habilidades sociais podem ser consideradas como operantes nas interações sociais, com probabilidade de produzir reforçadores positivos e negativos (BOLSONI-SILVA, 2007) e gerando sentimentos de bem-estar por trazer prazer ou por reduzir desprazer. Habilidades sociais educativas podem ser entendidas como aquelas voltadas propositalmente para o desenvolvimento e para aprendizagem de outra pessoa (DEL PRETTE; DEL PRETTE, 2001; CIA; PEREIRA; DEL PRETTE; DEL PRETTE, 2007). Dessa forma, especificamente para a interação estabelecida entre pais e filhos, Habilidades Sociais Educativas Parentais compõem-se do conjunto de habilidades sociais dos pais aplicáveis à prática educativa dos filhos, que podem estar presentes em seus repertórios isoladamente ou envolvidas com outras habilidades (BOLSONI-SILVA, 2008).

Ainda que existam aproximações quanto a definições de habilidades sociais educativas (DEL PRETTE; DEL PRETTE, 2009) e habilidades sociais educativas parentais (HSE-P; BOLSONI-SILVA, 2009) encontram-se na literatura dois sistemas de categorias, um descrito por Del Prette e Del Prette (2008) e outro por Bolsoni-Silva e Loureiro (2010). Del Prette e Del Prette (2008) propõem categorias de análises, passíveis de observação direta e de testagem empírica, elaboradas a partir da revisão de pesquisas da área. 
Bolsoni-Silva e Loureiro (2010) aprimoraram um sistema de categorias proposto por Bolsoni-Silva (2009) tendo por base o relato espontâneo de mais de 200 mães, as quais foram entrevistadas a partir de um roteiro de entrevista (RE-HSE-P):

1) comunicação (conversar, perguntar); 2) expressão de sentimentos e enfrentamento (expressar sentimentos positivos, negativos e opiniões, demonstrar carinho, brincar) e 3) estabelecimento de limites (identificar e consequenciar comportamentos socialmente habilidosos e não habilidosos, estabelecer regras, ter consistência, cumprir promessas, identificar erros e pedir desculpas). Outra característica desse sistema de categorias refere ao fato dessas habilidades sociais educativas serem investigadas contingentes aos comportamentos dos filhos (habilidades sociais e problemas de comportamento). O RE-HSE-P também permite mensurar práticas negativas de educação e variáveis de contexto. As categorias citadas podem ser organizadas em HSE-P, habilidades sociais infantis e variáveis de contexto (Características positivas da interação social) e práticas negativas de educação e problemas de comportamento (Características negativas da interação social), cujos escores permitem identificar comportamentos clínicos de não clínicos tendo o CBCL como padrão ouro e, portanto, como nota de corte.

Enquanto os problemas de comportamento parecem ser diretamente relacionados à quantidade de fatores de risco, como comentado anteriormente, essas habilidades sociais educativas parentais parecem minimizar os efeitos da punição funcionando como fatores de proteção, as quais sendo aprendidas podem assumir o papel de "saltos" comportamentais (BOLSONI-SILVA; MARTURANO, 2006) permitindo que a criança expanda seu repertório e aumente a chance de obter reforçamento. Supõe-se, então, levando-se em consideração os achados de Cia et al. (2007), que pessoas com um repertório mais elaborado de habilidades sociais estão sujeitas a efeitos negativos menos críticos nas situações adversas, pois parecem conseguir manter relações satisfatórias com outras pessoas.

De fato, a ligação entre práticas educativas e o manejo inefetivo familiar e escolar com problemas de comportamento merece ser destacada, pois qualquer modificação efetiva e duradoura dos comportamentos da criança pressupõe uma mudança na forma como os cuidadores lidam com ela (PINHEIRO et al., 2006). Sendo assim, é importante que exista a intervenção junto aos pais, para que as contingências se alterem, alterando consequentemente 0 comportamento do filho. Nesse ínterim, os pais podem assumir o papel de co-terapeutas, aprendendo a oferecer modelos e a modelar respostas socialmente habilidosas de seus filhos (BOLSONI-SILVA; SILVEIRA; MARTURANO, 2008).

Em estudo de revisão, Bolsoni-Silva, Villas Boas, Romera e Silveira (2010) analisaram 192 resumos de artigos publicados entre 1986 e 
junho de 2006, indexados em bases de dados, que avaliavam intervenções com foco em problemas de comportamento de crianças e adolescentes. Verificaram que existem muitos estudos que avaliam procedimentos de intervenção com pais, que têm como alvo habilidades como: comunicação, manejo de comportamentos, suporte social, conflitos e resolução de problemas, enfrentamento, autocontrole, promoção do relacionamento positivo e da competência social da criança e escuta ativa. As autoras destacam que a maior parte das pesquisas de intervenção priorizam a redução de problemas de comportamento e de práticas negativas de educação, sendo poucos os estudos que têm por foco promover o desenvolvimento da competência social dos pais e dos filhos. Bolsoni-Silva et. al. (2010) também sinalizam que existem poucos trabalhos onde os procedimentos estão claramente descritos, para que se viabilize a possibilidade de replicação e a condução de novos estudos. BolsoniSilva et al. (2010) concluem que procedimentos de intervenção com pais combinados com o trabalho com seus filhos podem favorecer a aprendizagem de repertórios mais amplos e considerados socialmente mais adequados, de forma que as intervenções poderiam se preocupar, além da alteração das práticas parentais dos pais, promover de forma direta ou indireta a competência social dos filhos. Em busca do aprimoramento e da maximização dos benefícios destes Programas, tanto para os pais/ cuidadores, como para os filhos, a curto, médio e longo prazo, há uma série de estudos, sobretudo na literatura internacional pertinente, que comparam procedimentos de intervenção. Há, por exemplo, pesquisas que comparam os efeitos de diferentes tipos de procedimentos de intervenção entre programas behavioristas e não behavioristas (LUNDAHL; RISSER; LOVEJOY, 2006) para modificação de comportamentos perturbadores infantis, isolando-se variáveis dependentes visadas pelo treinamento parental. Por outro lado, existem autores preocupados em promover interações positivas, além das que envolvem manejo comportamental, como nos diversos estudos encontrados referentes ao programa conhecido como Triplo-P - Programa Parental Positivo (SANDERS, 1999; SANDERS; MARKIE-DADDS; TULLY; BOR, 2000; BOR; SANDERS; MARKIE-DADDS, 2001; SANDERS; TULLY; TURNER; MAHER; MCAULIFFE, 2003).

Apesar de existirem diversas publicações na área de treinamento de pais e problemas de comportamento, poucas se dedicaram a avaliar diferentes procedimentos de intervenção. De maneira resumida e esquemática os estudos podem ser assim organizados: (a) intervenção comportamental familiar, reforçada, padrão, auto-dirigida e grupo controle (BOR; SANDERS; MARKIE-DADDS, 2002; SANDERS et al., 2000); (b) resolução de problemas colaborativa em comparação com o treinamento parental e programa de aprendizagem social baseada no treinamento dos pais ou um sistema 
de terapia familiar (GREENE et. al., 2004); (c) tratamento individualmente administrado de vídeo-modelagem, grupo de discussão desse tratamento, terapia direcionada em grupo e lista de espera (WEBSTER-STRATTON; KOLPACOFF; HOLLINSWORTH, 1988); (d) grupo de treinamento parental, treinamento da criança, grupo combinado de treinamento de crianças e de pais, e grupo controle (WEBSTER-STRATTON; HAMMOND, 1997); (e) treinamento dos pais, treinamento da criança e de professores (WEBSTER-STRATTON; REID; HAMMOND, 2004); (f) intervenção domiciliar $x$ intervenção clínica (HUTCHINGS; LANE; KELLY, 2004); (g) programas preventivos para crianças pré-escolares de maior duração e intensidade com de menor duração e menos intensos (NELSON; WESTHUE; MACLEOD, 2003).

Foram encontrados apenas três estudos, no que se refere à variável tempo (HUTCHINGS; LANE; KELLY, 2004; NELSON; WESTHUE; MACLEOD, 2003; SANDERS et al., 2000), os quais fazem menção a tal variável. No estudo de Sanders et al. (2000) houve dois tipos de intervenção: 1) a intervenção padrão que contava com 12 sessões de uma hora e; 2) a intervenção reforçada com 11 sessões de 60 a 90 minutos. Os resultados indicaram que as crianças no programa de intervenção reforçada obtiveram significativa melhora em relação a crianças submetidas à condições adversas. Entretanto, para os autores o resultado não dizia respeito ao tempo de intervenção.

No trabalho de Hutchings, Lane e Kelly (2004) um tratamento padrão e um mais intensivo, ao qual três sessões de cinco horas cada eram incluídas, foram comparadas. $O$ tratamento padrão era essencialmente comportamental, voltado a conselhos para os pais, tal como ensinar a reforçar comportamentos adequados e incluía psiquiatras e psicólogos clínicos infantis, além de outros especialistas. O tratamento intensivo diferia, principalmente, na inclusão de três sessões de cinco horas na Unidade de Tratamento, durante a qual os pais viam a eles mesmos e seus filhos em vídeo, recebiam um retorno e também novas estratégias de gestão. Um confortável ambiente doméstico e equipamentos necessários para a coleta de dados eram características essenciais na unidade, onde ocorreu uma observação direta por câmeras, espelhos e bug no ouvido, para que o terapeuta pudesse dar conselhos aos pais sobre o que fazer, elogiar e encorajar a novas estratégias de comportamento diante de certas situações. A partir dessa situação controlada, a generalização ao ambiente familiar era cuidadosamente apoiada e monitorada e o objetivo era garantir o sucesso parental na utilização de novas estratégias. Os autores acreditam que os resultados positivos do tratamento intensivo podem ser atribuídos não somente às horas a mais, mas também ao tipo de tratamento realizado na Unidade. Nelson, Westhue e MacLeod (2003), ao contrário dos outros dois estudos, concluíram que os programas de maior duração e 
intensidade atingiram melhores efeitos e estes permaneceram à longo prazo.

Entretanto, conclui-se, a partir da revisão bibliográfica realizada, que a literatura encontrada nessa área é reduzida e os resultados encontrados não são conclusivos, justificando novas pesquisas. Questiona-se então, se o tempo seria uma variável relevante nos resultados das intervenções; se existiriam diferenças significativas entre dois procedimentos, que se alteram apenas no tempo em que são ministrados.

A partir da revisão, acima mencionada, pode-se supor duas hipóteses: (a) que procedimentos mais rápidos podem ser mais intensos e com baixas taxas de desistência do tratamento, devido à estratégia de maior freqüência de contato com o programa de intervenção (BERGEL; GOUVEIA, 2005) e também poderia haver maior possibilidade do estabelecimento de uma aliança entre terapeuta e participante (ROBBINS; TURNER; ALEXANDER; PEREZ, 2003; ROBBINS; LIDDLE; TURNER; DAKOF; ALEXANDER; KOGAN, 2006); (b) por outro lado, a intervenção mais longa pode proporcionar um melhor acompanhamento dos participantes e um tempo maior para consolidar mudanças efetivas nos comportamentos (HUTCHINGS; LANE; KELLY, 2004).

Com base no exposto, o presente estudo objetivou comparar dois procedimentos de intervenção com pais que buscaram atendimento com queixas de dificuldades de relacionamento com seus filhos. Esses procedimentos diferiram apenas em relação ao tempo em que foram ministrados, sendo que o mesmo número de sessões foi aplicado apenas uma vez por semana a um grupo (G1) e duas vezes por semana a outro grupo (G2).

\section{Método}

\subsection{Participantes}

Participaram da intervenção 26 pais/cuidadores, os quais se inscreveram em um programa de intervenção com pais no Centro de Psicologia Aplicada de uma Universidade no interior paulista, em uma clínica-escola. Esses pais apresentavam queixas de dificuldades de interação - como dificuldades em conversar com os filhos, expressar opiniões ou sentimentos, impor limites e regras, manejar comportamentos inadequados, monitorar e consequenciar respostas - além de queixas de comportamentos inadequados dos filhos como agressividade e desobediência.

A intervenção foi realizada em 20 encontros, com pequenos subgrupos, de três a quatro pessoas cada, que, para esse estudo, foram reunidas em dois grupos de acordo com a frequência da 
intervenção. No primeiro (G1) a intervenção foi aplicada a 12 cuidadores primários apenas uma vez por semana, ao longo de um ano letivo; já o segundo grupo (G2) era composto por 14 cuidadores primários que passaram pelo tratamento duas vezes por semana, totalizando um semestre letivo.

Dos participantes de $\mathrm{Gl}$ dois eram pais e dez eram mães, das crianças e jovens seis eram do sexo masculino, cinco do sexo feminino e para uma a mãe não respondeu questões relativas ao gênero, idade e escolaridade do(a) filho(a). As idades dos filhos dos participantes do G1 variaram de um ano e sete meses a 18 anos ( média $=9,3$ anos; DP $=4,67$ ), sendo que três frequentavam 0 Ensino Infantil, sete o Ensino Fundamental e uma o Ensino Médio. Já em G2 havia quatro pais, nove mães e uma avó, sendo que os filhos e neto dos participantes eram, em sua maioria, do sexo feminino (nove pessoas), e cinco eram do sexo masculino. Desses, quatro pessoas cursavam o Ensino Infantil, oito o Ensino Fundamental e duas o Ensino Médio, sendo que uma já o completou. As crianças e jovens do G2 tinham a idade mínima de um ano e nove meses e a máxima de 21 anos e dez meses (média $=10,5$ anos; DP $=6,00$ ).

\section{Instrumento}

Utilizou-se, para avaliações de pré e pós-teste, o Roteiro de Entrevista de Habilidades Sociais Educativas Parentais (RE-HSE-P BOLSONI-SILVA; LOUREIRO, 2010), que investiga as habilidades sociais educativas parentais, frequência de ocorrência e variáveis antecedentes e consequente. As perguntas são feitas oralmente e as respostas espontâneas são registradas em um protocolo. Para cada questão da Entrevista há três opções em relação à periodicidade de ocorrência do comportamento: frequentemente, algumas vezes e quase nunca ou nunca. Se o participante escolher a primeira ou a segunda opção, são feitas outras perguntas relacionadas a características do comportamento referentes ao(a) filho(a) que mais motivou o atendimento.

A última versão do RE-HSE-P possui 13 conjuntos de perguntas das quais cinco prevêem a avaliação da frequência comportamental. São seis categorias - habilidade social educativa parental (hse-p), prática educativa negativa, habilidade social infantil (hs), problema de comportamento, variáveis de contexto e frequência comportamental positiva e negativa - e 70 opções previstas de respostas. Das cinco questões envolvidas na categoria frequência comportamental, três são relativas à frequência comportamental negativa: frequência de que os filhos fazem coisas que os pais não gostam; da dificuldade dos pais cumprirem promessas; e de expressão de sentimentos negativos. E duas questões referem-se à frequência comportamental 
positiva: frequência de concordância parental e; com que o pai faz perguntas sobre sexualidade. As outras categorias englobam situações, motivos, assuntos, sentimento dos pais, comportamento e reação dos pais e dos filhos e a reação do cônjuge, distribuídas nos temas das questões do instrumento.

Para análise do comportamento dos participantes, há questões sobre sexualidade, estabelecimento de limites, expressão de sentimentos positivos e negativos, expressão de carinho, situações em que o filho faz coisas que os pais aprovam ou desaprovam, identificação de erros e relacionamento conjugal. Para estas questões, a categoria relacionada à habilidade social educativa parental (hse-p) envolve comportamentos de comunicação e negociação, expressão de sentimentos e enfrentamento, sensação de bem estar, e também específicos à relação conjugal, como aceitar, concordar, pedir desculpas, entre outros. E a categoria práticas educativas negativas está relacionada a comportamentos não-habilidosos ativos e passivos, e sensação de mal estar. Com relação aos comportamentos dos filhos ou neto dos participantes, existem duas categorias específicas, para perguntas com os seguintes temas: conversa, estabelecimento de limites, expressão de opiniões e sentimento negativo, demonstração de carinho, comportamentos que os pais aprovam ou desaprovam, perguntas e dúvidas sobre sexualidade. A categoria habilidade social infantil (hs) envolve comportamentos habilidosos: disponibilidade social e cooperação ou expressão de sentimentos e enfrentamento. Retratando os aspectos considerados negativos do relacionamento, na categoria problemas de comportamento existem problemas de comportamento infantis externalizantes, internalizantes e outros comportamentos problema, como o descuido com ambiente e com as próprias coisas. E há também a categoria que diz respeito às variáveis contextuais, que está distribuída na maioria das questões do RE-HSE-P, e relacionamse a temas diversos, diversos momentos do dia, situações diversas, situações de lazer, horário de refeição, concepções de certo e errado, motivos para estabelecer limites, problemas pessoais dos pais, e questões do filho sobre relacionamento sexual e doenças sexualmente transmissíveis. (BOLSONI-SILVA; LOUREIRO, 2010).

\section{Caracterização da intervenção}

O procedimento foi ministrado por estudantes do último ano da Graduação em Psicologia e/ou por psicólogos recém formados, em um total de 20 sessões para cada grupo. Nelas abordou-se habilidades sociais básicas no relacionamento dos participantes com seus filhos, propondo tarefas de casa, discussões sobre os tópicos das 
sessões e treinamento de repertórios de comportamentos associados ao tema em questão.

Os temas trabalhados baseavam-se nas habilidades de comunicação, como iniciar e manter conversação, fazer e responder perguntas; expressão de sentimentos positivos (elogiar, dar e receber feedback positivo, agradecer) e negativos (dar e receber feedback negativo); expressar e ouvir opiniões de concordância ou discordância; fazer e recusar pedidos e conhecimento de direitos humanos básicos. Também foram trabalhadas as diferenças entre comportamentos habilidoso, não habilidoso ativo e não habilidoso passivo; a capacidade de lidar com críticas, admitir os próprios erros e pedir desculpas. O estabelecimento de limites é um dos temas essenciais também explorados, assim como o observar, discriminar as contingências que influenciam para que os filhos se comportem de forma "desejada" e "indesejada", questionar regras parentais sobre o que é certo e errado fazer, ignorar comportamentos "indesejados", consequências comportamentos "desejados", dar atenção, expressar afeto, solicitar mudança, dar regras, consequenciar regras e negociar. Esse procedimento é resultado de pesquisas de intervenção conduzidas previamente e que demonstraram sua efetividade (BOLSONI-SILVA; SILVEIRA; RIBEIRO, 2008; BOLSONI-SILVA, SILVEIRA; MARTURANO, 2008).

No Grupo 1 a intervenção em ocorreu por aproximadamente cinco meses espaçado no decorrer do ano letivo, seguindo o seguinte cronograma:

- Março: Início da seleção dos participantes;

- Abril: Condução de entrevistas iniciais para avaliação diagnóstica (quatro sessões, uma por semana) das pessoas interessadas (medidas de pré-teste). Observa-se que para fins desta pesquisa está sendo utilizado apenas o RE-HSE-P;

- Maio a junho: Realização de oito sessões de grupo e uma sessão individual com cada participante para avaliação do atendimento do semestre;

- Julho: Recesso escolar e suspensão do atendimento;

- Agosto a outubro: Realização de doze sessões restantes;

. Novembro: Aplicação dos instrumentos de pós-teste.

Para o Grupo 2 a intervenção em grupo teve duração de aproximadamente três meses, contando também com a avaliação de pré-teste e de pós-teste, sendo um mês cada uma delas, perfazendo o total de atendimento de cinco meses, iniciado em março do ano letivo e terminado em julho do mesmo ano. 


\section{Procedimentos de coleta e análise de dados}

O primeiro passo do procedimento, após a seleção dos candidatos, foi a realização da entrevista individual com cada um dos participantes, na qual receberam informações sobre os objetivos da intervenção, puderam esclarecer eventuais dúvidas e assinaram o Termo de Consentimento Pós-I nformado garantindo o sigilo e confidencialidade dos dados por parte dos pesquisadores e o direito de abandonar 0 procedimento a qualquer momento, sem prejuízos. Nessa entrevista foi realizado o pré-teste e o pós-teste aconteceu em um encontro individual após o término da intervenção. Todos os dados mencionados são referentes aos filhos e ao neto que mais motivaram o participante da intervenção ao atendimento. Considerando a diversidade do repertório comportamental dos participantes dos grupos, optou-se por comparar o desempenho dos participantes dos Grupos 1 e 2 durante o procedimento de intervenção, pela análise qualitativa, com a intenção de identificar contingências entrelaçadas entre os comportamentos dos pais e seus filhos. O RE-HSE-P permite classificar os escores obtidos por cada participante nas principais categorias do RE-HSE-P, em clínicos (C) e não clínicos (NC), obtidos a partir de curva ROC. Para cada uma das seis categorias do RE-HSE-P, existe um escore ou um intervalo considerado limítrofe, definindo os escores considerados clínicos ou não clínicos para os aspectos positivos e negativos da interação estabelecida entre pais e filhos.

As categorias que englobam aspectos positivos da interação são as habilidades sociais educativas parentais (hse-p), habilidades sociais infantis (hs criança), as variáveis de contexto e o total positivo que é a soma dos escores obtidos nas categorias habilidades sociais educativas parentais, habilidades sociais da criança, variáveis de contexto e frequência positiva. As categorias que tratam dos aspectos negativos do relacionamento são as práticas negativas, os problemas de comportamento e o total negativo - que consiste na média da soma das categorias expressão de sentimentos negativos, problemas de comportamento e frequência negativa. Primeiramente, os escores dos participantes dos dois grupos, de pré e pós-testes, foram classificados clínicos e não clínicos, de acordo com curva ROC do estudo de Bolsoni-Silva e Loureiro (2010). Posteriormente foi realizada a análise qualitativa dos escores médios das questões que compõem o RE-HSEP, também para os Grupos 1 e 2, no pré e póstestes.

\section{Resultados}

Para a classificação clínica e não clínica considerou-se escores de aspectos positivos e negativos da interação, para os Grupos 1 e 2. As 
Tabelas 1 e 2 representam o número de participantes classificados com escores clínicos e não clínicos, ao início e ao término da intervenção e também o número de participantes que tiveram sua classificação mantida ou alterada. Com os aspectos negativos, ao contrário, ocorre abaixo do escore limítrofe. Os escores são considerados não clínicos, e acima ou iguais o escore limítrofe são considerados clínicos.

Em ambas as tabelas, as linhas descrita como "Início" e "Término" contêm o número de participantes que obtiveram escore clínico (C) ou não clínico (NC) nos Grupos 1 e 2. Na linha "Alterações", as iniciais " $\mathrm{C}$ - NC" indicam o número de participantes que passou do escore clínico (C) no pré-teste ao não clínico (NC) no pós; e ao contrário representam as iniciais "NC - C" que apontam o número de participantes que iniciou a intervenção com escore não clínico (NC) e finalizou com escore clínico (C). E a linha "Manutenções" demonstra o número de participantes dos dois grupos que permaneceram com a mesma classificação ( $\mathrm{C}$ ou NC) no início e no término da intervenção.

A seguir, a Tabela 1 menciona tanto aspectos positivos quanto negativos do relacionamento, retratando as seguintes categorias: habilidades sociais educativas, práticas negativas, habilidades sociais infantis e problemas de comportamento.

Tabela 1 - Quantidade de participantes dos Grupos 1 e 2 que obtiveram escores clínicos (C) e não clínicos (NC) nas categorias hse$\mathrm{p}$, práticas negativas, hs e problemas de comportamento.

\begin{tabular}{|c|c|c|c|c|c|c|c|c|c|}
\hline \multirow{4}{*}{\multicolumn{2}{|c|}{ Aspectos do relacionamento }} & \multicolumn{4}{|c|}{ Pais/ cuidadores } & \multicolumn{4}{|c|}{ Filhos/ Neto } \\
\hline & & \multirow{2}{*}{\multicolumn{2}{|c|}{ HSEP }} & \multirow{2}{*}{\multicolumn{2}{|c|}{$\begin{array}{l}\text { Práticas } \\
\text { negativas }\end{array}$}} & \multirow{2}{*}{\multicolumn{2}{|c|}{ HS }} & \multirow{2}{*}{\multicolumn{2}{|c|}{$\begin{array}{l}\text { Problemas } \\
\text { de compor- } \\
\text { tamento }\end{array}$}} \\
\hline & & & & & & & & & \\
\hline & & $\mathrm{G} 1^{1}$ & $\mathrm{G} 2^{2}$ & $\mathrm{G} 1^{1}$ & $\mathrm{G} 2^{2}$ & $\mathrm{G} 1^{1}$ & $\mathrm{G}^{2}{ }^{2}$ & $\mathrm{G} 1^{1}$ & $\mathrm{G} 2^{2}$ \\
\hline \multirow[t]{2}{*}{ Início } & $\mathrm{C}$ & 8 & 9 & 3 & 5 & 10 & 11 & 8 & 9 \\
\hline & NC & 4 & 5 & 9 & 9 & 2 & 3 & 4 & 5 \\
\hline \multirow[t]{2}{*}{ Alterações } & $C-N C$ & 3 & 1 & 3 & 4 & 4 & 2 & 2 & 5 \\
\hline & $N C-C$ & 1 & 4 & 1 & 0 & 0 & 2 & 2 & 3 \\
\hline \multirow[t]{2}{*}{ Manutenções } & $\mathrm{C}$ & 5 & 8 & 0 & 1 & 6 & 9 & 6 & 4 \\
\hline & NC & 3 & 1 & 8 & 9 & 2 & 1 & 2 & 2 \\
\hline \multirow[t]{2}{*}{ Término } & C & 6 & 12 & 1 & 1 & 6 & 11 & 8 & 7 \\
\hline & NC & 6 & 2 & 11 & 13 & 6 & 3 & 4 & 7 \\
\hline
\end{tabular}

Observa-se, na Tabela 1, que para as habilidades sociais educativas (hse-p), os dois grupos apresentaram semelhanças no pré-teste. 0 
Grupo 1 iniciou a intervenção com oito de doze pessoas com escore clínico, enquanto o Grupo 2, composto por quatorze participantes, contou com nove pessoas com escore clínico. Três pessoas do Grupo 1 e uma pessoa do Grupo 2 passaram do escore clínico para o não clínico; do Grupo 1, uma pessoa apresentou escore não clínico no pré-teste e no pós-teste obteve escore clínico, enquanto no Grupo 2 foram quatro pessoas. Cinco pessoas do Grupo 1 mantiveram escore clínico desde o início e três pessoas mantiveram o escore não clínico, enquanto no Grupo 2, oito pessoas apresentaram escore clínico no pré e no pós-teste e uma pessoa manteve desde o início escore não clínico. Ao término da intervenção, os resultados foram diferentes: o Grupo 1 mostrou melhor desempenho, passando a ter metade de seus participantes com escore clínico, e metade com escore não clínico; enquanto no Grupo 2, doze pessoas apresentaram escore clínico e apenas duas que apresentaram escore não clínico.

Em relação às práticas negativas, três participantes do Grupo 1 iniciaram a intervenção com escore clínico e nove participantes com escore não clínico. Do Grupo 2, cinco pessoas iniciaram a intervenção com escore clínico e nove com escore não clínico. No pós-teste, para o Grupo 1, as três pessoas que apresentaram escore clínico no préteste, apresentaram escore não clínico, porém, uma pessoa que apresentara escore não clínico, passou a apresentar no pós-teste escore clínico. E para o Grupo 2, houve quatro superações. Sendo assim, ao término da intervenção, houve resultados positivos para os dois grupos, pois apenas uma pessoa de cada grupo, apresentou escore clínico.

Para as habilidades sociais infantis (hs), percebe-se que dez participantes do Grupo 1 iniciaram a intervenção com escore clínico, e dois participantes com escore não clínico; e do Grupo 2, onze participantes apresentaram escore clínico no pré-teste, e três apresentaram escore não clínico. Após a intervenção, para o Grupo 1 houve quatro superações, ou seja, quatro pessoas passaram do escore clínico ao não clínico; e para o Grupo 2 houve duas superações e duas quedas igualando o percentual de pessoas do grupo com escore clínico e não clínico do pré-teste. Considerando-se as manutenções do escore do pré ao pós-teste, do Grupo 1 houve seis pessoas que permaneceram com escore clínico e duas com escore não clínico, e no Grupo 2, nove pessoas mantiveram com escore clínico e uma com escore não clínico. Desta forma, no pósteste o Grupo 1 obteve melhor desempenho, já que seis pessoas apresentaram escore clínico, e seis pessoas escore não clínico; e do Grupo 2, como no pré-teste, onze pessoas apresentaram escore clínico e três escore não clínico.

Afirmar que o Grupo 2 não obteve progressos para as habilidades sociais infantis, baseando-se apenas no dados numéricos seria um engano, já que as pessoas que apresentaram escore clínico e não 
clínico no pré e no pós-teste foram diferentes. Ressalta-se que a população que apresentou mudanças, no caso os filhos dos participantes, não recebeu intervenção direta, e sim foi atingida através de alterações no modo como os pais se comportaram, o que atesta a modificação do comportamento e a generalização para o ambiente natural, indicando os benefícios trazidos pelo do procedimento.

Considerando os problemas de comportamento, os Grupos 1 e 2 (Tabela 1) iniciaram a intervenção com proporções parecidas: respectivamente, oito (G1) e nove (G2) pessoas com escore clínico, e quatro (G1) e cinco (G2) pessoas com escore não clínico. Após a intervenção houve duas superações e duas quedas no Grupo 1, e cinco superações e três quedas no Grupo 2. Então, no pós-teste, oito participantes do Grupo 1 apresentaram escore clínico e quatro escore não clínico; e o Grupo 2, mostrou melhor desempenho, já que metade dos participantes apresentou escore clínico, e a outra metade escore não clínico.

A seguir, a Tabela 2 ilustra a quantidade de participantes dos Grupos 1 e 2 que obtiveram escore clínico ou não clínico no decorrer do processo de intervenção, em relação às variáveis de contexto e aos totais negativo e positivo.

Tabela 2 - Quantidade de participantes dos Grupos 1 e 2 que obtiveram escores clínicos (C) e não clínicos (NC) nas variáveis de contexto, total negativo e total positivo.

\begin{tabular}{llcccccc}
\hline Aspectos do relacionamento & \multicolumn{2}{c}{ Contexto } & \multicolumn{2}{c}{ Total negativo } & \multicolumn{2}{c}{ Total Positivo } \\
& & $\mathrm{G} 1^{1}$ & $\mathrm{G} 2^{2}$ & $\mathrm{G} 1^{1}$ & $\mathrm{G} 2^{2}$ & $\mathrm{G} 1^{1}$ & $\mathrm{G} 2^{2}$ \\
\hline Início & $\mathrm{C}$ & 3 & 1 & 10 & 13 & 6 & 6 \\
\multirow{2}{*}{ Alterações } & $\mathrm{NC}$ & 9 & 13 & 2 & 1 & 6 & 8 \\
& $\mathrm{C}-\mathrm{NC}$ & 3 & 1 & 4 & 6 & 3 & 3 \\
Manutenções & $\mathrm{NC}-\mathrm{C}$ & 0 & 1 & 1 & 1 & 0 & 1 \\
& $\mathrm{C}$ & 0 & 0 & 6 & 7 & 3 & 3 \\
Término & $\mathrm{NC}$ & 9 & 12 & 1 & 0 & 6 & 7 \\
& $\mathrm{C}$ & 0 & 1 & 7 & 8 & 3 & 4 \\
& $\mathrm{NC}$ & 12 & 13 & 5 & 6 & 9 & 10 \\
${ }^{1}$ G1 é compó composto por 12 participantes; & & & & & &
\end{tabular}

As variáveis de contexto constituem um elemento importante para a promoção de interações positivas dos pais com seus filhos. Sendo assim, nota-se que, de acordo com a Tabela 2, três pessoas do Grupo 1 iniciaram a intervenção com escore clínico, e nove com escore não 
clínico; e uma pessoa do Grupo 2 apresentou escore clínico no préteste, e treze apresentaram escores não clínicos. Observa-se que os dois grupos já contavam com um bom repertório para variáveis de contexto antes mesmo da intervenção, e ainda assim houve resultados positivos para ambos, pois todos os participantes do Grupo 1 apresentaram escore não clínico no pós-teste, e o Grupo 2 também superou as dificuldades iniciais, mas surgiram outras para um dos participantes que passou a apresentar escore clínico no pós-teste.

Em relação ao total positivo, metade dos participantes do Grupo 1 apresentaram, no pré-teste, escore clínico, e metade apresentou escore não clínico. Dos participantes do Grupo 2, seis apresentaram escore clínico e oito escore não clínico. Houve, para o Grupo 1, três superações; e para o Grupo 2, três superações e uma queda. Desta forma, ao término da intervenção ambos tiveram resultados positivos: o Grupo 1 terminou a intervenção com três pessoas com escore clínico e nove com escore não-clínico; e o Grupo 2 com quatro pessoas com escore clínico e onze com escore não clínico.

Para o total negativo, dez participantes do Grupo 1 apresentaram escore clínico no pré-teste e dois participantes apresentaram escore não clínico; e treze participantes do Grupo 2 apresentaram escore clínico e um apresentou escore não clínico. Houve quatro superações e uma queda no Grupo 1, e seis superações e uma queda no Grupo 2. Assim os Grupos 1 e 2 terminaram a intervenção, respectivamente com sete (G1) e oito (G2) pessoas com e escore clínico, e cinco (G1) e seis (G2) participantes com escore não clínico. Tanto para o total positivo quanto para o negativo, observam-se semelhanças no préteste e resultados positivos para os dois grupos em ambos.

\section{Discussão}

Com a intenção de avaliar a variável tempo em procedimentos de intervenção de treinamento de habilidades sociais com grupos de pais, os resultados de pré e pós-testes foram analisados. A análise qualitativa com a classificação em escores obtidos no RE-HSE-P em clínicos e não clínicos possibilitou a análise do desempenho de cada participante e, consequentemente, dos grupos cuja intervenção foi aplicada em uma vez por semana (G1) e duas vezes por semana (G2).

De acordo com as análises realizadas, nota-se, primeiramente, que o Grupo 1, no qual a intervenção foi aplicada uma vez por semana, sendo assim no período mais longo, obteve melhora principalmente nas habilidades sociais educativas parentais e nas habilidades sociais infantis. Por outro lado, o Grupo 2, no qual a intervenção foi aplicada duas vezes por semana, realizada em um semestre letivo, obteve uma maior melhora na diminuição das práticas negativas e nos 
problemas de comportamento, com uma melhora menor das habilidades sociais. Assim, em ambos observa-se que uma população que não recebeu diretamente a intervenção foi atingida: os filhos e neto dos participantes - os quais no Grupo 1 aumentaram suas habilidades sociais e no Grupo 2 diminuíram os problemas de comportamento, de acordo com a intervenção de seus pais sobre seus comportamentos.

Não é possível afirmar que o Grupo 1 obteve, em geral, um melhor resultado, pois ambos os grupos poderiam apresentar os dois resultados, já que a diminuição das práticas negativas pode estar diretamente relacionada com a promoção de habilidades sociais. Desta forma, pode-se colocar a hipótese de que aprender habilidades sociais educativas parentais pode ser mais difícil e demandar mais tempo para a consolidação no repertório comportamental e indicamse as interdependências comportamentais onde o comportamento dos pais é ambiente ou consequente para o comportamento dos filhos e vice-versa. Assim, pais habilidosos têm maior probabilidade de que os filhos aprendam, por imitação ou por modelagem habilidades sociais, contribuindo para sua competência social.

De maneira resumida, notou-se um melhor desempenho do Grupo 1 em relação às habilidades sociais educativas parentais e das habilidades sociais infantis. Por outro lado, o Grupo 2 obteve desempenho melhor para os problemas de comportamento. E tanto o Grupo 1 quanto o Grupo 2 mostraram resultados positivos em relação às práticas negativas, variáveis de contexto e totais positivo e negativo.

Portanto, de acordo com os resultados obtidos nas análises realizadas, a hipótese colocada anteriormente, de que a intervenção mais longa pode proporcionar um melhor acompanhamento dos participantes e um tempo maior para consolidar mudanças efetivas nos comportamento (HUTCHINGS; LANE; KELLY, 2004), parece se confirmar. E os resultados assemelharam-se aos de Nelson, Westhue e MacLeod (2003), em que os melhores efeitos vieram de programas de maior duração e intensidade. Assim, é possível concordar com Lohr, Pereira, Andrade e Kirchner (2007) quando apontam que para a determinação da qualidade de um programa, a duração da intervenção e a frequência das atividades são variáveis importantes e devem ser cuidadosamente delimitadas.

De acordo com Feitosa et al. (2005) pode-se afirmar que a solução de problemas de comportamento depende do emprego de medidas socioeducativas para desenvolver habilidades sociais para socialização. Sendo assim, a melhoria observada nas habilidades sociais educativas do Grupo 1 está diretamente relacionada com o aumento de habilidades sociais das crianças. Da mesma forma, a diminuição dos problemas de comportamento pode estar relacionada com a redução das práticas negativas no caso do Grupo 2. 
Minimizando-se os déficits ou excessos comportamentais, as crianças podem ter mais fácil acesso a novas contingências de reforçamento, adquirindo repertórios relevantes na aprendizagem (BOLSONI-SILVA; DEL PRETTE, 2003). E se a quantidade de fatores de risco e os problemas de comportamento são diretamente proporcionais, percebe-se que reduzindo as práticas negativas, diminuem-se também os problemas de comportamento (BOLSONI-SILVA; MARTURANO, 2006). Diminuindo os déficits de habilidades sociais dos pais para manejar os comportamentos dos filhos, pode-se evitar o surgimento ou manutenção dos comportamentos considerados inadequados (PINHEIRO et al., 2006).

Ao longo dessa análise, percebe-se que, do pré ao pós-teste, alguns escores, para parte da amostra, antes não clínicos, passaram a ser clínicos, como por exemplo: (a) para ambos os grupos, nas categorias habilidades sociais educativas parentais, problemas de comportamento e total negativo; (b) somente para Grupo 1, na categoria práticas negativas; (c) e para o Grupo 2, nas categorias habilidades sociais infantis, variáveis de contexto e total positivo. Dessa forma, parte dos resultados indica que algumas pessoas que eram inicialmente habilidosas passaram a não ser após a intervenção. Uma hipótese explicativa é a de que após a intervenção os participantes tornaram-se mais críticos em relação à avaliação de seu próprio comportamento. Assim, o participante classificaria seu comportamento após a intervenção desconsiderando seu repertório inicial e tomando como modelo o que ele gostaria de fazer em tais situações, após autoconhecimento (SKINNER, 1974/1995) do que se espera do desempenho deles na interação com os filhos, o aprenderam após a intervenção. De acordo com Skinner (1979/2000), o autoconhecimento é fundamental para o monitoramento dos próprios comportamentos e para o autocontrole, consequentemente a pessoa é mais hábil a emitir respostas que aumentem a probabilidade de obtenção de reforçadores.

Para testar se a passagem dos escores de não clínicos para clínicos significam diminuições dos desempenhos sociais dos participantes, poderia se aplicar os mesmos instrumentos de pré e pós-testes, algumas sessões após o início da intervenção. Nesse teste, os participantes já conheceriam alguns princípios básicos de habilidades sociais e poderiam ser capazes de avaliar com mais propriedades seu repertório.

Outro fator que pode estar influenciando os resultados é a não homogeneidade das populações. O estudo de Bolsoni-Silva, Silveira e Ribeiro (2008) indica que os resultados melhoram quando a amostra é homogênea e com maiores indicativos de problemas de comportamento. As autoras avaliaram três participantes, duas mães e uma avó, que pretendiam melhorar seu relacionamento, respectivamente, com seus filhos e neta, através do treinamento de 
habilidades sociais educativas parentais. Participaram de um procedimento semelhante ao descrito no presente trabalho e os resultados apontaram que o efeito mais expressivo de aquisição de habilidades sociais foi de uma participante, cujas avaliações anteriores à intervenção apontaram para critérios clínicos ou limítrofes em todas as escalas de avaliação. As outras duas participantes que não faziam parte de uma população clínica e sim preventiva, não obtiveram resultados tão importantes. (BOLSONISILVA; SILVEIRA; RIBEIRO, 2008).

É importante destacar que a aquisição de habilidades sociais, de acordo com Del Prette e Del Prette (2009), envolve um processo de aprendizagem contínuo, e talvez por este motivo, o grupo que recebeu a intervenção mais longa (G1), possa ter obtido melhora significativa nas categorias habilidades sociais educativas parentais e habilidades sociais infantis. Isso pode indicar que a melhora nos problemas de comportamento já apresentada por G2 poderia ser ampliada em conjunto com as habilidades sociais, se houvesse um período maior de intervenção.

O desenvolvimento das habilidades varia de acordo com as demandas próprias do estágio de desenvolvimento do indivíduo e de seu repertório atual, considerando o efeito que causa sobre o ambiente. Bolsoni-Silva (2007) aponta que as habilidades sociais são consideradas operantes nas interações, na abordagem da Análise do Comportamento, pois há probabilidade de produzirem reforçadores positivos e negativos de forma a ampliar o repertório de comportamentos socialmente adequados, trazendo consequências positivas para os indivíduos e relações satisfatórias com outras pessoas.

O trabalho combinado com pais e filhos pode propiciar melhores resultados, uma vez que os filhos também podem aprender por modelação ou modelagem a comportar-se de forma considerada socialmente adequada, servindo como antecedentes ou consequentes para o comportamento dos pais. Dessa forma, seria possível o fortalecimento e a potencialização do treinamento com os pais, pois aumentaria a probabilidade de obter consequências reforçadoras para ambos (BOLSONI-SILVA et al., 2010).

Concluindo, existiam comportamentos considerados clínicos para os dois grupos, mas nenhum dos grupos pode ser considerado clínico para todos os comportamentos avaliados e também porque as pessoas que buscaram os grupos de intervenção não estavam aguardando atendimento em listas de espera do Centro de Psicologia Aplicada da Universidade em questão. As pessoas chegaram aos grupos através de anúncios e cartazes e é esta população de maior parte heterogênea que geralmente busca uma clínica escola. Dessa forma, destaca-se a importância de procedimentos de intervenção de 
prevenção e promoção que atuem junto à comunidade, antes que as pessoas busquem espontaneamente por atendimento.

Assim, observou-se que é possível trabalhar para prevenir problemas de comportamento e práticas negativas, quando os comportamentos que trazem mais punição que reforçadores ainda não estejam fortemente instalados nos repertórios das crianças e dos pais, e também para promover interações positivas e competência social. Analisar a possibilidade de novas práticas parentais pode indicar mudanças nas práticas culturais de forma a garantir mais reforçadores positivos e promover interações positivas entre pais/cuidadores e seus filhos.

A hipótese inicial de que a intervenção mais longa poderia proporcionar um tempo maior para a consolidação de mudanças efetivas nos comportamentos (HUTCHINGS; LANE; KELLY, 2004) é confirmada, já que o grupo em que a intervenção foi ministrada uma vez por semana (G1) obteve ganhos em habilidades sociais. E mesmo que exista em procedimentos mais rápidos, maior possibilidade de aliança (ROBBINS; TURNER; ALEXANDER; PEREZ, 2003; ROBBINS; LIDDLE et al., 2006), parece não ter sido uma variável determinante para influenciar nos resultados.

\section{Considerações finais}

As limitações que surgiram, no decorrer deste estudo, foram o baixo número de participantes e o fato de a amostra não ser homogênea, contando com diferentes características dos participantes, tanto do Grupo 1 quanto do Grupo 2, como por exemplo, a grande variação de idade dos filhos dos participantes, com escolaridade desde o Ensino Infantil até Ensino Médio completo. Esses fatores dificultariam uma tentativa de análise estatística, já que não se poderia afirmar que os grupos eram equivalentes. Os resultados aqui considerados, bem como as análises subsequentes, não podem ser generalizados para outras populações, já que cada caso deve ser estudado particularmente e detalhadamente, considerando o número reduzido de participantes e a influência de diferentes variáveis nos resultados de uma pesquisa como esta.

O fato de o repertório comportamental infantil ter melhorado apenas em um dos grupos é de difícil explicação. Uma forma de reduzir essa dificuldade em outras pesquisas seria a utilização de outros instrumentos de coleta de dados do repertório infantil, tanto na forma de relato e escalas, quanto na forma de observação direta no ambiente natural e/ou artificial.

Justifica-se, então, a contribuição deste estudo para a área da Psicologia, em razão das lacunas existentes na literatura pertinente, 
no que diz respeito especialmente a comparação do tempo das intervenções.

Os resultados positivos para ambos os grupos que participaram da pesquisa, chamaram a atenção, com especial destaque para a expressiva melhora de habilidades sociais no Grupo 1, o qual participou da intervenção apenas uma vez por semana.

Ambos os procedimentos contaram com o formato semi-estruturado embora tenha sido considerados as características individuais dos participantes -, e de curto prazo - indicando que são intervenções que podem ser aplicadas em escolas e também em universidades. Neste sentido, este tipo de pesquisa pode colaborar na implantação de uma política pública que venha minimizar problemas de comportamento nas relações parentais e escolares. Assim sendo, espera-se que as pesquisas futuras possam ampliar a amostra, torná-la homogênea, a fim de combinar a observação direta, as medidas processuais, além das de relato e de pré e pós-teste.

\section{Referências}

BERGEL, F. S.; GOUVEIA, N. Retornos freqüentes como nova estratégia para adesão ao tratamento de tuberculose. Revista Saúde Pública, v. 39, n. 6, 2005, p. 898-905

BOLSONI-SILVA, A. T. Roteiro de Entrevista de Habilidades Sociais Educativas Parentais (RE-HSE-P): Categorias e testagem preliminares. In: WEBER, L. D. (Org.). Família e Desenvolvimento - Visões I nterdisciplinares, Curitiba: Juruá, 2007, p.145-158.

BOLSONI-SILVA, A. T.; DEL PRETTE, A. Problemas de comportamento: um panorama da área. Revista brasileira de terapia comportamental cognitivo, São Paulo, v. 5, n. 2, p. 91103, 2003.

BOLSONI-SILVA, A. T.; DEL PRETTE, A. O que os pais falam sobre suas habilidades sociais e a de seus filhos? Argumento, Jundiaí, v. 7, n. 3, p. 71-86, 2002.

BOLSONI-SILVA, A. T.; LOUREIRO, S. R. Estudos de validação e confiabilidade do Roteiro de Entrevista de Habilidades Sociais Educativas Parentais (RE-HSE-P). Revista Avaliação Psicológica, Itatiba, v. 9, n. 1, p. 63-75, 2010.

BOLSONI-SILVA, A. T.; MARTURANO, E. M. A qualidade da interação pais e filhos e sua relação com problemas de comportamento préescolares. In: BANDEIRA, M.; DEL PRETTE, Z. A. P.; DELL PRETTE, A. (Org.). Estudos sobre Habilidades Sociais e Relacionamento Interpessoal. São Paulo: Casa do Psicólogo, 2006, p. 89-104.

BOLSONI-SILVA, A. T.; PAIVA, M. M.; BARBOSA, C. G. Problemas de comportamento de crianças/adolescentes e dificuldades de 
pais/cuidadores: um estudo de caracterização. Psicologia Clínica, Rio de Janeiro, v. 21, n. 1, p. 169-184, 2009.

BOLSONI-SILVA, A. T.; SILVEIRA, F. F.; MARTURANO, E. M. Promovendo habilidades sociais educativas parentais na prevenção de problemas de comportamento. Revista brasileira de terapia comportamental cognitiva, São Paulo, v. 10, n. 2, p. 125-142, 2008.

BOLSONI-SILVA, A. T.; SILVEIRA, F. F.; RIBEIRO, D. C. Avaliação dos efeitos de uma intervenção com mães/cuidadoras: contribuições do treinamento em habilidades sociais. Contextos Clínicos, São Leopoldo, v. 1, n. 1, p. 19-27, 2008.

BOLSONI-SILVA, A. T.; VILLAS BOAS, A. C. V. B.; ROMERA, V. B.; SILVEIRA, F. F. Caracterização de programas de intervenção com crianças e/ou adolescentes. Arquivos Brasileiros de Psicologia, Rio de Janeiro, v. 62, n. 1, p. 104-115, 2010.

BOR, W.; SANDERS, M. R; MARKIE-DADDS, C. The effects of the Triple P-positive Parenting Program on preschool children with cooccurring disruptive behavior and attentional/hyperactive difficulties. J ournal-of-Abnormal-Child-Psychology, v. 30, n. 6, p. 571-587, 2002.

ClA, F.; PEREIRA, C. S.; DEL PRETTE, Z. A. P.; DEL PRETTE, A. Habilidades sociais das mães e envolvimento com os filhos: Um estudo correlacional. Estudos de Psicologia, Campinas, v. 24, n. 1, p. 03-11, 2007.

DEL PRETTE, A.; DEL PRETTE, Z. A. P. Psicologia das relações interpessoais: vivências para 0 trabalho em grupo. Petrópolis: Vozes, 2001.

DEL PRETTE, Z. A. P.; DEL PRETTE, A. Psicologia das habilidades sociais: terapia e educação. Petrópolis: Vozes, 1999.

DEL PRETTE, Z. A. P.; DEL PRETTE, A. Psicologia das habilidades sociais: diversidade teórica e suas implicações. Petrópolis: Vozes, 2009.

FEITOSA, F. B.; MATOS, M. G.; DEL PRETTE, Z. A. P.; DEL PRETTE, A. Suporte social, nível socioeconômico e o ajustamento social e escolar de adolescentes portugueses. Temas em Psicologia, Ribeirão Preto, v.13, n. 2, p. 129-138, 2005.

GREENE, R. W.; ABLON, J. S.; GORING, J. C.; RAEZER-BLAKELY, L.; MARKEY, J.; MONUTEAUX, M. C.; HENIN, A.; EDWARDS, G.; RABBITT, S. Effectiveness of collaborative problem solving in affectively dysregulated children with oppositional-defiant disorder: initial findings. Journal of Consulting and Clinical Psychology, Washington, v. 72, n. 6, p. 1157-1164, 2004.

HUTCHINGS, J.; LANE, E.; KELLY, J. Comparison of Two Treatments for Children with Severely Disruptive Behaviours: A Four-Year Followup. Behavioural and Cognitive Psychotherapy, New York, v. 32, n. 1, p. 15-30, 2004. 
LOHR, S. S.; PEREIRA, A. C. S.; ANDRADE, A. L. M.; KIRCHNER, L. F. Avaliação de programas preventivos: relato de experiência. Psicologia em Estudo, Maringá, v. 12, n. 3, p. 641-649, 2007. LUNDAHL, B.; RISSER, H. J.; LOVEJOY, M. C. A meta-analysis of parent training: Moderators and follow-up effects. Clinical Psychology Review, Oklahoma, v. 26, n. 1, p. 86-104, 2006.

NELSON, G.; WESTHUES, A.; MACLEOD, J. A meta-analysis of longitudinal research on preschool prevention programs for children. Prevention \& Treatment, lowa, v. 6, n. 1, 2003.

PINHEIRO, M. I. S.; HAASE, V. G.; DEL PRETTE, A.; AMARANTE, C. L. D.; DEL PRETTE, Z. A. P. Treinamento de habilidades sociais educativas para pais de crianças com problemas de comportamento. Psicologia Reflexão e Crítica, Porto Alegre, v. 19, n. 3, p. 407414, 2006.

ROBBINS, M. S.; LIDDLE, H. A.; TURNER, C. W.; DAKOF, G. A.; ALEXANDER, J. F.; KOGAN, S. M. Adolescent and Parent Therapeutic Alliances as Predictors of Dropout in Multidimensional Family Therapy. J ournal of Family Psychology, New York, v. 20, n. 1, p. 108-116, 2006.

ROBBINS, M. S.; TURNER, C. W.; ALEXANDER, J. F.; PEREZ, G. A. Alliance and Dropout in Family Therapy for Adolescents With Behavior Problems: Individual and Systemic Effects. Journal of Family Psychology, New York, v. 17, n. 4, p. 534-544, 2003.

SANDERS, M. R. Triple P-Positive Parenting Program: towards an empirically validated multilevel parenting and family support strategy for the prevention of behavior and emotional problems in children. Clinical Child Family Psychology Review, New York, v. 2, n. 2, p. 71-90, 1999.

SANDERS, M. R.; MARKIE-DADDS, C.; TULLY, L. A.; BOR, W. The Triple P-Positive Parenting Program: A comparison of enhanced, standard, and self-directed behavioral family intervention for parents of children with early onset conduct problems. Journal of Consulting and Clinical Psychology, Washington, v. 68, n. 4, p. 624-640, 2000.

SANDERS, M. R.; TULLY, L. A.; TURNER, K. M.; MAHER, C.; MCAULIFFE, C. Training GPs in parent consultation skills. An evaluation of training for the Triple P-Positive Parenting Program. Australian Family Physician, Kansas, v. 32, n. 9, p. 763-768, 2003.

SIDMAN, M. Coerção e suas implicações. Trad. ANDERY, M. A.; SÉRIO, T. M. Campinas: Editorial Psy, 1989/1995.

SKINNER, B. F. Ciência e Comportamento Humano. São Paulo: Martins Fontes, 1979/ 2000.

SKINNER, B. F. Sobre o Behaviorismo. São Paulo: Cultrix, $1974 / 1995$. 
WEBSTER-STRATTON, C.; HAMMOND, M. Treating children with earlyonset conduct problems: a comparison of child and parent training interventions. Journal of Consulting and Clinical Psychology, Washington, v. 65, n. 1, p. 93-109, 1997.

WEBSTER-STRATTON, C.; KOLPACOFF, M.; HOLLINSWORTH, T. Selfadministered videotape therapy for families with conduct-problem children: Comparison with two cost-effective treatments and a control group. Journal of Consulting and Clinical-Psychology, Washington, v. 56, n. 4, p. 558-566, 1988.

WEBSTER-STRATTON, C.; REID, M. J.; HAMMOND, M. Treating Children With Early-Onset Conduct Problems: Intervention Outcomes for Parent, Child, and Teacher Training. Journal of Clinical Child and Adolescent Psychology, New York, v. 33, n. 1, p. 105-124, 2004.

WELLS, K. C.; EGAN, J. Social learning and systems family therapy for childhood oppositional disorder: Comparative treatment outcome. Comprehensive Psychiatry, Kansas, v. 29, n. 2, p. 138-146, 1988.

\section{Endereço para correspondência \\ Alessandra Turini Bolsoni-Silva}

Av. Engenheiro Luiz Edmundo Carrijo Coube, 14-01, Vargem Limpa, CEP 17033360, Bauru - SP, Brasil

Endereço eletrônico: bolsoni@fc.unesp.br

Laura Moreira Borelli

Av. Engenheiro Luiz Edmundo Carrijo Coube, 14-01, Vargem Limpa, CEP 17033360, Bauru - SP, Brasil

Endereço eletrônico: Imborelli@yahoo.com.br

Recebido em: 06/07/2010

Reformulado em: 21/10/2010

Aceito para publicação em: 21/10/2010

Acompanhamento do processo editorial: Adriana Benevides Soares

\section{Notas}

* Professora da Faculdade de Ciências da Universidade Estadual Paulista-UNESP, Bauru-SP, Brasil; Livre Docente, Pós- doutorado, Bolsista Produtividade CNPq.

** Graduada em Psicologia na Universidade Estadual Paulista-UNESP.

${ }^{1}$ Este trabalho foi financiado pela Fapesp (processo no. 2008/03912-2) enquanto projeto de Iniciação Científica da segunda autora sob orientação da primeira. 